\section{Sintomatología depresiva y calidad de vida en estudiantes de medicina en alta latitud sur}

\author{
CAREN ALVARADO-ARAVENA ${ }^{1, \mathrm{a}}$, CLAUDIA ESTRADA-GOIC ${ }^{2, \mathrm{~b}}$, \\ CRISTIAN NÚÑEZ-ESPINOSA ${ }^{1, \mathrm{c}}$
}

\section{Depression and seasonal sensitivity among medical students residing in high southern latitudes}

Background: There is no evidence for the association of depressive symptoms with the sense of well-being of undergraduate medical students residing in high southern latitudes (HSL). Aim: To assess the frequency of depressive symptoms and its relationship with seasonal sensitivity and quality of life in medical students residing in HSL. Material and Methods: The Beck anxiety and depressive symptoms inventory, the seasonal pattern assessment questionnaire and the quality-of-life questionnaire of the WHO were applied to 102 medical students residing in Magallanes, Chile. Results: Fifty-two percent of respondents reported some degree of depressive symptoms. These symptoms were significantly associated with the seasonal pattern score $(r=0.432, p<0.01)$. They were also inversely associated with parameters of physical health and psychosocial health ( $r=-0.567, p<0.01$ and $r=-0.708, p<0.01$, respectively). There was also a correlation between depressive and anxiety symptoms. Conclusions: Depressive symptoms are common in medical students residing in HSL and they are associated with seasonal sensitivity and quality of life measures.

(Rev Med Chile 2021; 149: 357-365)

Key words: Depression; Quality of Life; Seasonal Affective Disorder; Students, Medical.

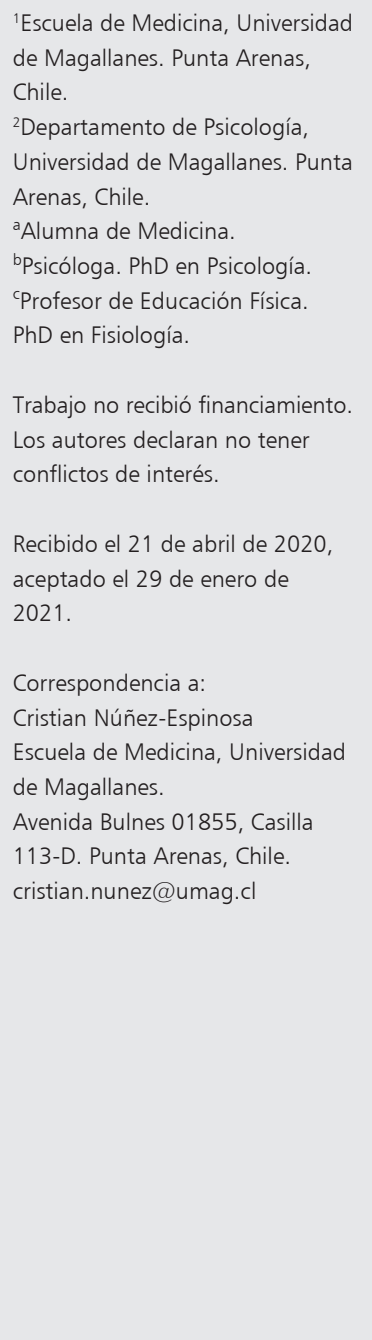

${ }^{1}$ Escuela de Medicina, Universidad de Magallanes. Punta Arenas, Chile.

2Departamento de Psicología, Universidad de Magallanes. Punta Arenas, Chile.

umna de Medicina. ción Física.

Trabajo no recibió financiamiento. Los autores declaran no tener

Recibido el 21 de abril de 2020 aceptado el 29 de enero de Cristian Núñez-Espinosa Escuela de Medicina, Universidad de Magallanes. 113-D. Punta Arenas, Chile. cristian.nunez@umag.cl

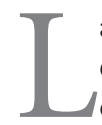

a depresión es una alteración patológica del estado de ánimo, caracterizada por un des$\checkmark$ censo del humor, que termina en tristeza y es acompañada de diversos síntomas y signos ${ }^{1}$. En Chile, la sintomatología depresiva alcanza 17,2\% en personas mayores de 15 años, llegando a 25,7\% en mujeres ${ }^{2}$. En la población universitaria se ha observado que las tasas de depresión son mayores que en la población general de la misma edad ${ }^{3-5} y$ que, en carreras demandantes a nivel académico, como medicina, posibilitan aun más el deterioro de la salud mental, presentando altas prevalencias de depresión ${ }^{6,7}$, pudiendo llegar hasta el suicidio ${ }^{8,9}$. Además, estudios realizados en Brasil, China y
Chile señalan que estos estudiantes presentan menor calidad de vida que otros jóvenes, principalmente en relación a los parámetros de salud psicológica y relaciones sociales ${ }^{10-12}$.

En zonas de alta latitud se ha observado que los cambios respecto al tiempo de exposición a luz solar pueden incidir en alteraciones patológicas del estado de ánimo, lo que ha sido descrito como trastorno afectivo estacional (TAE) $)^{13,14}$ y su variable subclínica denominada sensibilidad estacional ${ }^{15,16}$. Ambas formas de vulnerabilidad psicobiológica se asocian a los cambios de insolación en las diferentes estaciones del año, es decir, una variable atmosférica que tiene impacto en el 
funcionamiento biopsicosocial de los individuos ${ }^{17}$, pudiendo alterar el orden biológico, psicológico o social presentándose en estaciones con mayor o menor insolación ${ }^{18}$. Este tipo de patología estacional se manifiesta en alteraciones fisiopatológicas ${ }^{19}$, las cuales se expresan a través de alteraciones en el estado de ánimo, principalmente sintomatología depresiva acompañada de síntomas atípicos, entre los cuales se distinguen el aumento del apetito, la hipersomnia, el aumento de peso y un deseo sexual disminuido ${ }^{15}$. Entonces, ambos fenómenos, el TAE y la sensibilidad estacional, caracterizados por la presencia de cambios cíclicos del estado de ánimo, pueden ser condicionante o agravante del estado depresivo de los estudiantes ${ }^{17,18}$, pudiendo percibirse tanto en invierno como en verano, considerándose en este caso un patrón mixto ${ }^{20}$.

Sabemos que la prevalencia de sensibilidad estacional en la población general aumenta en latitudes más altas y disminuye cerca del ecuador ${ }^{21}$. Es más común en invierno, en mujeres en edad fértil y la edad media de aparición ronda los 20-35 años $^{18,19}$. Estos últimos criterios coinciden con la etapa de formación académica universitaria, por lo que proponemos como hipótesis que estudiar en regiones de altas latitudes, por su correlación con los cambios más extremos de insolación entre estaciones, puede condicionar la aparición de estas afecciones psicológicas que impactan directamente la salud mental de las personas ${ }^{22-24}$.

A pesar de la importancia de ampliar el conocimiento de la sintomatología depresiva y su impacto en el bienestar de estudiantes, no existen estudios en latitud sur que incluyan población universitaria, por lo que este estudio tiene como objetivo conocer la prevalencia de sintomatología depresiva y su relación con la sensibilidad estacional y calidad de vida de los estudiantes de medicina que residen en alta latitud sur (ALS).

\section{Materiales y Participantes}

\section{Participantes}

Participaron estudiantes de medicina de la Universidad de Magallanes, en Punta Arenas, Chile, ubicada en latitud $53^{\circ}$ sur. Se defina alta latitud sur desde la latitud $50^{\circ}$ hasta el Polo Sur. En total, 102 estudiantes de medicina participaron en este estudio. Para el criterio de inclusión se aplicó ser mayor de edad, cursar entre primero y quinto año de la carrera de medicina, no presentar ninguna afectación psicológica clínicamente diagnosticada y residir de forma permanente al menos 10 meses al año en ALS. La estimación del tamaño de la muestra se calculó con un nivel de confianza de $95 \%$ y un $\alpha \leq 0,05$. Para el análisis se construyeron cuatro grupos según el tiempo de estadía en la región: G1, hasta 18 meses en la región; G2, entre 19 y 36 meses; G3, 37 meses y más; G4, nativos de la región. El estudio fue aprobado por el Comité de Ética de Investigación de la Universidad de Magallanes y se realizó de acuerdo con la Declaración de Helsinki sobre los principios éticos en seres humanos.

\section{Instrumentos}

Información sociodemográfica: Mediante anamnesis, se registró el género del participante, edad, ciudad de origen, tiempo de estadía en la región y presencia o antecedentes de enfermedades psicológicas. La permanencia en la región de ALS fue encuestada a cada estudiante y comprobada con el registro de asistencia a clases.

Sintomatología depresiva: Se utilizó el Inventario de Depresión de Beck (BDI-II), el cual es un instrumento de autoaplicación de 21 ítems, con cuatro opciones de respuesta tipo escala Likert, con valores desde el 0 al 3 . Cada sujeto debe referir su estado de ánimo en las últimas dos semanas, logrando un puntaje máximo de 63 puntos $^{25}$.

Sintomatología ansiosa: Se utilizó el Inventario de Ansiedad de Beck (BAI), traducido al español por Sanz y Navarro ${ }^{26}$. El instrumento se aplica como autoinforme por medio de 21 ítems, con cuatro opciones de respuesta en escala Likert valoradas desde 0 a 3 . Se discrimina la sintomatológica ansiosa clínicamente significativa con un puntaje de corte de 16 puntos ${ }^{27}$.

Sensibilidad estacional: Se aplicó el Cuestionario de Evaluación del Perfil Estacional (SPAQ, por sus siglas en inglés $)^{28}$, el cual es una escala autoadministrada y de aplicación atemporal, compuesto por 6 ítems que miden la variación estacional del estado de ánimo durante los meses del año. Este test origina un índice general de puntuación estacional (SSI), indicando la presencia de TAE, que refleja un cuadro depresivo con un patrón estacional (PE); la forma más leve de TAE ha sido considerada como un subsíndrome (TAE-S), denominada en inglés coloquial winter blues ${ }^{29}$. Otro ítem evalúa el grado de gravedad, determinando 
si los cambios estacionales son considerados un problema.

Calidad de vida: Se utilizó el World Health Organization Quality of Life Questionnaire (WHOQoL-BREF) $)^{30}$, el cual es una versión reducida del WHOQoL- $100^{31}$. Este cuestionario autoadministrado y transcultural, creado por la Organización Mundial de la Salud (OMS), determina la calidad de vida percibida por la persona en las últimas dos semanas, evaluando cuatro dominios: Salud física (SF), relacionada con dolor y molestia, grados de energía, fatiga, sueño y descanso, movilidad, actividades cotidianas, necesidad de medicamentos y capacidad para trabajar; salud psicológica (SP), que involucra sentimientos positivos, pensamiento y memoria, autoestima, imagen corporal, sentimientos negativos y espiritualidad; salud social (SS), que abarca las relaciones personales, soporte social y actividad sexual; salud ambiental (SA), la cual refleja seguridad, condiciones de vivienda, finanzas, servicio de salud, acceso a información, actividades placenteras, ambiente físico y el transporte ${ }^{31}$.

\section{Procedimiento}

Los participantes firmaron voluntariamente su consentimiento informado, para luego responder en una sola sesión los instrumentos detallados. Estos fueron autoaplicados durante el invierno, ya que la evaluación de presencia de síntomas es más directa en este momento (horas de luz promedio invierno: 2,8; verano: 7,4). Cada evaluación se programó en un tiempo libre de 30 min para responder todas las preguntas.

\section{Análisis estadístico}

Para el análisis estadístico se utilizó el paquete SPSS. Se realizaron análisis descriptivos y correlacionales ( $\mathrm{r}$ Sperman), análisis de Kruskal-Wallis y U Mann-Whitney. Las regresiones simples se confeccionaron a partir de valores absolutos. Todos los análisis se realizaron con una significancia estadística menor a 0,05 .

\section{Resultados}

\section{Caracterización de estudiantes encuestados}

El tamaño de la muestra fue de 102 estudiantes de medicina (edad: $21 \pm 2,5$ ), de los cuales 54,9\% eran mujeres y $45,1 \%$ hombres. El 29,4\% de estu- diantes tenía residencia permanente en ALS y 70, $6 \%$ estudiantes venía de otras regiones de menor latitud y residía en la zona por motivos de estudio. En relación a los grupos de permanencia en ALS, G1 estuvo compuesto por $24,5 \%$ de la muestra, G2 por $23,5 \%$, G3 por $22,5 \%$ y G4 por $29,4 \%$ de los estudiantes.

\section{Sintomatología de depresión}

Se evaluó la sintomatología depresiva a través de BDI-II, aplicándose cuatro criterios para comparar los grupos: depresión mínima (puntaje entre 0-13), depresión leve (puntaje entre 14-19), depresión moderada (puntaje entre 20-28) y depresión grave (puntaje entre 29-63) ${ }^{20}$. El 52\% de los encuestados presentó algún nivel de depresión, mientras que $48 \%$ solo presentó depresión mínima, la cual no se considera depresión clínica. La comparación rangos-promedios de las variables en función del nivel de depresión de sujeto mostró significancia estadística con BAI, sensibilidad estacional y los dominios de WHOQoL-BREF, no así el género del sujeto y su tiempo de permanencia en la región (Tabla 1). Para conocer la relación absoluta de las variables, se correlacionó la sintomatología depresiva con los factores del estudio, pudiendo apreciarse su significancia en la Tabla 2.

\section{Sensibilidad estacional}

En relación a la prevalencia de sensibilidad estacional, según el SSI, 65,7\% tenía TAE, 19,6\% presentaba winter blues y $14,7 \%$ tenía un puntaje promedio de normalidad. En cuanto al patrón de estacionalidad, $52 \%$ de los estudiantes presentaba ambos patrones estacionales, $28,4 \%$ presentaba patrón de verano y $2 \%$ mostró un patrón de invierno exclusivo, mientras que $17,6 \%$ restante tenía ausencia de patrones estacionales. Por último, según grado de gravedad, 51,5\% de los estudiantes consideró que estas variaciones estacionales eran un problema y $48,5 \%$ no lo consideraba un problema. Se observaron correlaciones positivas entre el tiempo de permanencia en la región y el SSI $\left(r_{(\mathrm{n}=102)}=0,29, p=0,03\right)$, y entre el SSI y la sintomatología depresiva de los estudiantes $\left(r_{(\mathrm{n}=102)}=0,43, p=0,01\right)$.

\section{Sintomatología ansiosa}

La sintomatología ansiosa se correlacionó positivamente con la ansiedad detectada $\left(\mathrm{r}_{\mathrm{s}(\mathrm{n}=102)}=0,44, \mathrm{p}=0,001\right)$ y negativamente con 
Depresión en estudiantes de medicina en alta latitud sur - C. Alvarado-Aravena et al

Tabla 1. Rangos promedios para cada una de los niveles de depresión de BAI

\begin{tabular}{|lccccccc|}
\hline & $\begin{array}{c}\text { Depresión } \\
\text { mínima } \\
(\mathbf{n}=\mathbf{4 9 )}\end{array}$ & $\begin{array}{c}\text { Depresión } \\
\text { leve } \\
(\mathbf{n}=\mathbf{2 2})\end{array}$ & $\begin{array}{c}\text { Depresión } \\
\text { moderada } \\
(\mathbf{n}=\mathbf{1 5})\end{array}$ & $\begin{array}{c}\text { Depresión } \\
\text { grave } \\
(\mathbf{n}=\mathbf{1 6})\end{array}$ & H & gl & p \\
\hline Sexo & 48,48 & 49,00 & 60,90 & 55,38 & 3,306 & 3 & 0,347 \\
\hline Tiempo de permanencia (TP) & 45,08 & 55,36 & 57,17 & 60,53 & 5,047 & 3 & 0,168 \\
\hline Ansiedad (BAI) & 44,08 & 49,09 & 54,10 & 75,09 & 25,659 & 3 & 0,000 \\
\hline Seasonal Score Index (SSI) & 39,50 & 64,25 & 57,40 & 65,19 & 22,898 & 3 & 0,000 \\
\hline Patrón estacional (PE) & 42,26 & 64,11 & 53,47 & 60,63 & 12,474 & 3 & 0,006 \\
\hline Salud física (SF) & 67,32 & 45,57 & 38,67 & 23,25 & 32,836 & 3 & 0,000 \\
\hline Salud psicológica (SP) & 69,73 & 51,39 & 33,37 & 12,81 & 52,725 & 3 & 0,000 \\
\hline Salud social (SS) & 58,73 & 52,14 & 40,20 & 39,06 & 8,099 & 3 & 0,044 \\
\hline Salud ambiental (SA) & 59,43 & 48,25 & 48,10 & 34,88 & 9,238 & 3 & 0,026 \\
\hline
\end{tabular}

Esta tabla muestra los rangos promedios para cada una de los niveles de depresión de BDI-II en relación a las variables estudiadas, según la prueba de Kruskal-Wallis. Los resultados están organizados según la siguiente nomenclatura: $\mathrm{H}$, valor estadístico; gl, grado de libertad; $p$, valor de significancia.

Tabla 2. Correlaciones entre los parámetros del estudio

\begin{tabular}{|c|c|c|c|c|c|c|c|c|c|c|}
\hline & & TP & SF & SP & SS & SA & BDI & BAI & PE & SSI \\
\hline \multirow[t]{2}{*}{ Tiempo de permanencia } & $r_{(\mathrm{s})}$ & --- & $-0,108$ & $-0,155$ & 0,026 & 0,110 & $0,221^{*}$ & $-0,044$ & 0,093 & $0,287^{* *}$ \\
\hline & $p$ & & 0,279 & 0,119 & 0,795 & 0,270 & 0,026 & 0,661 & 0,353 & 0,003 \\
\hline \multirow[t]{2}{*}{ Salud física (SF) } & $r_{(\mathrm{s})}$ & & --- & $0,629^{* *}$ & $0,500^{* *}$ & $0,574^{* *}$ & $-0,567^{* *}$ & $-0,423^{* *}$ & $-0,216^{*}$ & $-0,353^{* *}$ \\
\hline & $p$ & & & 0,000 & 0,000 & 0,000 & 0,000 & 0,000 & 0,029 & 0,000 \\
\hline \multirow[t]{2}{*}{ Salud psicológica (SP) } & $r_{(\mathrm{s})}$ & & & --- & $0,502^{* *}$ & $0,464^{* *}$ & $-0,708^{* *}$ & $-0,362^{* *}$ & $-0,178$ & $-0,338^{* *}$ \\
\hline & $p$ & & & & 0,000 & 0,000 & 0,000 & 0,000 & 0,074 & 0,001 \\
\hline \multirow[t]{2}{*}{ Salud social (SS) } & $r_{(s)}$ & & & & --- & $0,539^{* *}$ & $-0,275^{\star *}$ & $-0,328^{* *}$ & $-0,050$ & $-0,205^{*}$ \\
\hline & $p$ & & & & & 0,000 & 0,005 &, 001 & 0,619 & 0,039 \\
\hline \multirow[t]{2}{*}{ Salud ambiental (SA) } & $r_{(\mathrm{s})}$ & & & & & --- & $-0,291^{* *}$ & $-0,355^{* *}$ & $-0,218^{*}$ & $-0,141$ \\
\hline & $p$ & & & & & & 0,003 & 0,000 & 0,028 & 0,157 \\
\hline \multirow[t]{2}{*}{ Depresión (BDI) } & $r_{(s)}$ & & & & & & --- & $0,442^{* *}$ & $0,287^{* *}$ & $0,432^{* *}$ \\
\hline & $p$ & & & & & & & 0,000 & 0,003 & 0,000 \\
\hline \multirow[t]{2}{*}{ Ansiedad (BAl) } & $r_{(s)}$ & & & & & & & --- & 0,146 & $0,243^{*}$ \\
\hline & $p$ & & & & & & & & 0,143 & 0,014 \\
\hline \multirow[t]{2}{*}{ Patrón de estacional (PE) } & $r_{(\mathrm{s})}$ & & & & & & & & --- & $0,428^{* *}$ \\
\hline & $p$ & & & & & & & & & 0,000 \\
\hline
\end{tabular}

Esta tabla muestra la correlación de los diferentes parámetros del estudio. Los resultados están organizados según la siguiente nomenclatura: TP, Tiempo de permanencia en alta latitud; SF, Salud física; SP, Salud psicológica; SS, Salud social; SA, Salud ambiental; BDI, Inventario de Depresión de Beck; BAI, Inventario de Ansiedad de Beck; PE, Patrón de estacionalidad; SSI, Seasonal Score Index. Los coeficientes de correlación de Spearman, ( $r$ y su significancia estadística están descritas ( $p$ valor). 


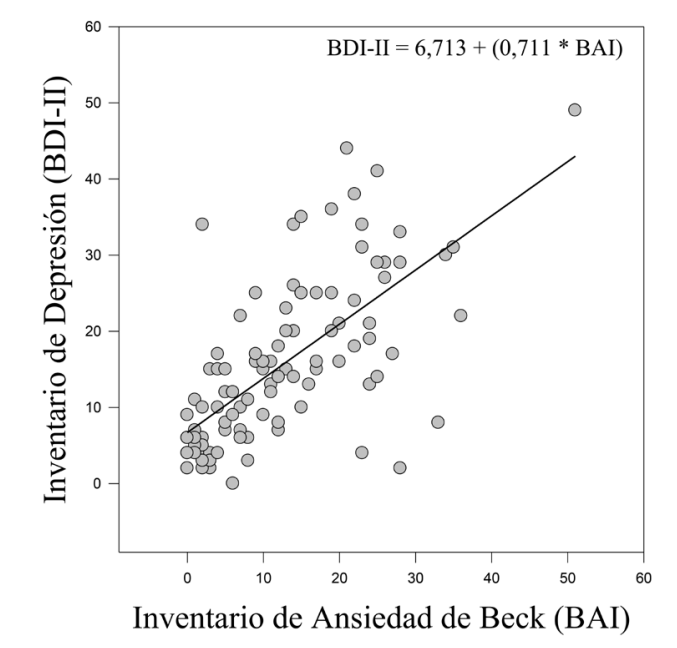

Figura 1. Modelo predictivo entre las variables depresión y ansiedad. todas las variables de WHOQoL-BREF. A través de una regresión lineal de valores absolutos, se obtuvo una significancia estadística para la creación de un modelo predictivo entre las variables depresión y ansiedad, la cual se observa en la Figura 1.

\section{Calidad de vida}

Los valores de percepción de calidad de vida según el grupo de estudio son presentados en la Figura 2. Se mostraron correlaciones negativas entre las diferentes escalas de WHOQoL-BREF en relación a depresión y ansiedad. SSI se correlacionó negativamente con SF $\left(r_{(\mathrm{n}=102)}=-0,20\right.$, $p=0,04)$, y SP $\left(r_{(\mathrm{n}=102)}=-0,20, p=0,04\right)$, ocurriendo lo mismo con el patrón estacional y salud física $\left(r_{(\mathrm{n}=102)}=-0,22, p=0,03\right)$, y salud ambiental $\left(r_{(\mathrm{n}=102)}=-0,22, p=0,03\right)$. En otros términos, cuando aumentó la percepción de sensibilidad

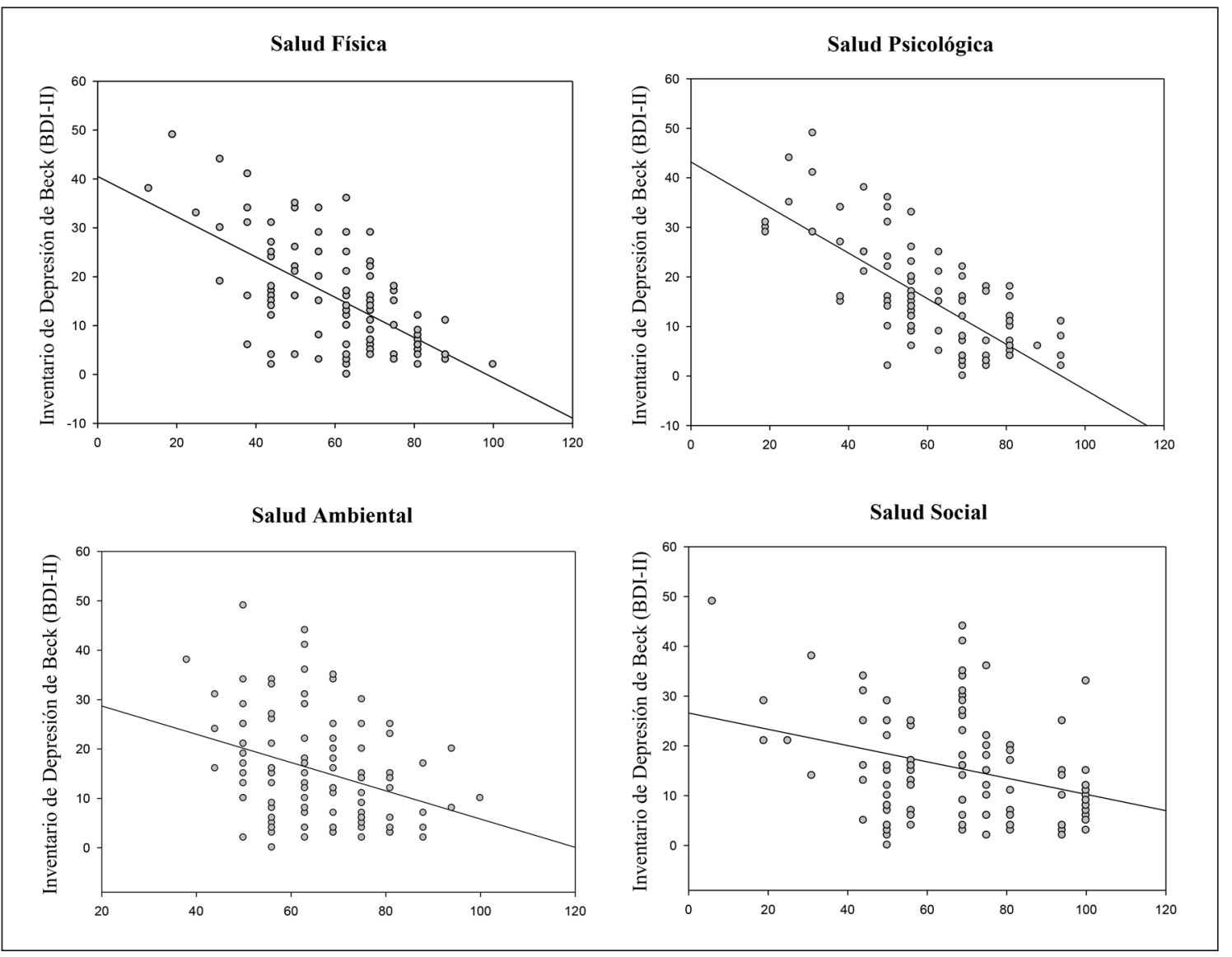

Figura 2. Distribución del puntaje total de BDI-II según los dominios de WHOQoL-BREF. 
Depresión en estudiantes de medicina en alta latitud sur - C. Alvarado-Aravena et al

Tabla 3. Medidas de los cuatro dominios de WHOQoL-Bref según grupos de estudio

\begin{tabular}{|lcccc|}
\hline & G1 (n = 25) & G2 (n = 24) & G3 (n = 23) & G4 (n = 30) \\
Salud física (SF) & $66,40 \pm 18,16^{\mathrm{a}}$ & $59,00 \pm 13,75$ & $53,17 \pm 19,07$ & $62,23 \pm 12,89$ \\
Salud psicológica (SP) & $64,60 \pm 15,71$ & $62,25 \pm 16,09$ & $55,22 \pm 18,64$ & $58,13 \pm 17,85$ \\
Salud social (SS) & $68,72 \pm 21,73$ & $66,21 \pm 24,18$ & $63,35 \pm 19,12$ & $70,03 \pm 17,79$ \\
Salud ambiental (SA) & $66,36 \pm 13,03$ & $63,00 \pm 11,35$ & $61,83 \pm 11,85^{\mathrm{b}}$ & $70,57 \pm 12,27$ \\
\hline
\end{tabular}

Esta tabla muestra la media \pm desviación estándar de los cuatro dominios de WHOQol-Bref según los diferentes grupos de estudio. Las diferencias significativas son descritas según el siguiente código: apara $p<0,05$ comparado con G3; ${ }^{b}$ para $p<0,05$ comparado G4.

estacional, disminuyeron salud física, salud psicológica y salud ambiental. Se analizó también cada dominio de WHOQoL-Bref en tiempo de permanencia en alta latitud, lo cual es presentado en la Tabla 3.

\section{Discusión}

Los resultados de este estudio, en relación a sintomatología depresiva de estudiantes universitarios, son similares a los encontrados en la literatura ${ }^{27-29}$. Aunque sabemos que los estudiantes de medicina tienden a manifestar mayor estrés, depresión y prevalencia de trastornos psiquiátri$\cos ^{8,30,31}$, los efectos de estudiar en altas latitudes no han sido investigados. La condicionante de latitud implica estudiar en climas extremos y exponerse a diferentes períodos de exposición a luz estacional durante el año, lo cual tiene una estrecha relación con la aparición de síntomas depresivos ${ }^{24,32}$. Aunque existe cierta controversia respecto a si es la latitud o los cambios de insolación los que generan cambios en las personas, ambos se correlaciona fuertemente y sus efectos son difíciles de separar ${ }^{22-24}$.

En este estudio, se muestra que 52\% de los alumnos reporta algún grado de sintomatología depresiva, existiendo una correlación positiva entre depresión, patrón estacional y SSI y, al comparar los rangos medios de las variables, se puede observar que el grupo de estudiantes con depresión leve tiene rangos altos en patrón estacional y SSI, especialmente al compararlos con el grupo de depresión mínima, los que no tienen depresión clínica. Estos hallazgos pueden ser interesantes, dado que a nivel de depresión leve puede ser un punto de inflexión en cuanto a la afectación de sensibilidad estacional, que sabemos que está muy ligada a una mala calidad de vida de la población ${ }^{33}$. A modo de hipótesis, podemos decir que la afectación de los primeros niveles de depresión puede ser un mayor condicionante de sensibilidad estacional, con lo cual se podría prevenir la afectación de salud por cambios de exposición a luz estacional a través de control de las primeras etapas de la depresión. En base al cuestionario SPAQ, también pudimos encontrar que los estudiantes tienen una alta prevalencia de algún tipo de patrón estacional $(82,4 \%)$, siendo la gran mayoría de ellos afectados tanto en invierno como en verano. La alta prevalencia de sensibilidad estacional, impensadamente, no es percibida como un problema importante por el $48,5 \%$ de los estudiantes, por lo que se presume que la escasez de información en relación a estas temáticas permite la falta de consideración de la sensibilidad estacional como una problemática al bienestar diario de las personas.

Estudios de calidad de vida en estudiantes de medicina comparan sensibilidad estacional de estudiantes residentes en media latitud con estudiantes provenientes de África, donde estos últimos presentaron mayor sensibilidad estacional ${ }^{34}$. En nuestro estudio, 70,5\% de estudiantes son originarios de otras zonas de menor latitud y los resultados muestran una correlación positiva con mayor índice de sensibilidad estacional. Resulta interesante que el tiempo de permanencia en alta latitud puede ser uno de los factores que influyen en los valores de SSI, tal como se muestra en la Figura 3, estimándose que a mayor tiempo de residencia en alta latitud, mayor es la sensibilidad estacional. 


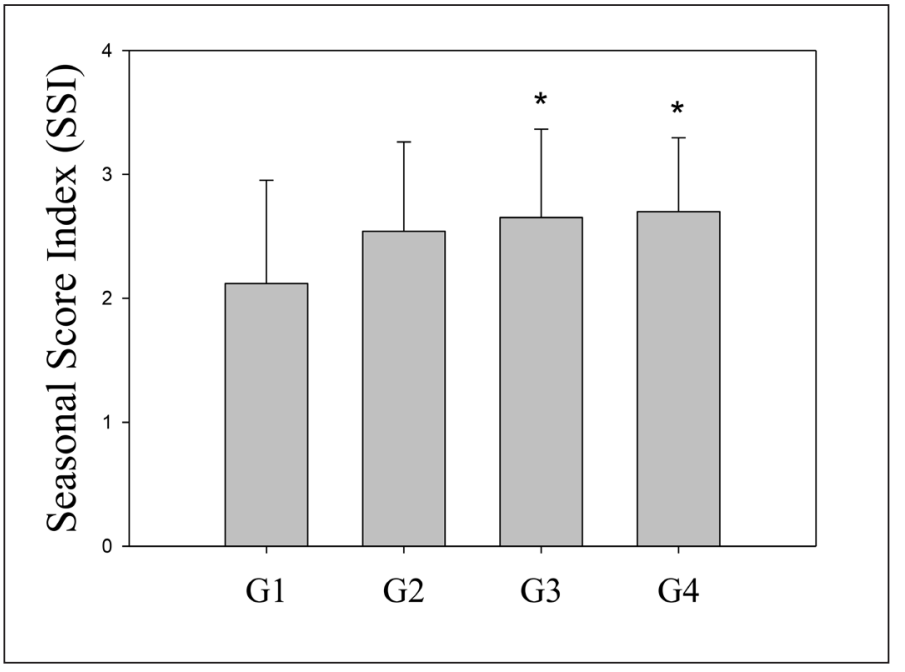

Figura 3. SSI para diferentes grupos de estudio. Diferencias significativas se describen acorde al siguiente código: *, para diferencias con G1, $p<0,05$.
En relación a la sintomatología ansiosa, los rangos medios de ansiedad se condicen con mayor nivel de depresión, pudiendo crear un modelo predictivo de ambas variables, permitiendo el posible manejo de síntomas depresivos a través de estrategias académicas que disminuyan conductas ansiosas de los estudiantes.

La salud física y salud psicológica se correlacionan negativamente con los síntomas depresivos. Los rangos medios de ambos dominios son muy altos en estudiantes que no presentan sintomatología depresiva clínica, disminuyendo en la medida que la depresión es más grave. Otro factor interesante que se encontró es que los estudiantes que llegan a vivir a altas latitudes, mientras más tiempo pasen en la zona, presentan menor dominio físico, especialmente comparando G1 y G3. No obstante, la diferencia solo se da con estudiantes de otras latitudes, ya que los residentes permanentes presentan valores inferiores que G1. Considerando que G3 es el grupo de mayor tiempo de permanencia en la región y que, por ende, está en cursos superiores de la carrera, ambas diferencias pueden relacionarse con el ingreso de los estudiantes a cursos superiores de área clínica, lo cual implica una disminución de tiempo libre y cambio de su ambiente físico de trabajo y de descanso. En el trabajo de Ibarra et al.$^{10}$ se comparan estudiantes provenientes de de distintas regiones de Chile con aquellos alumnos de la Región Metropolitana del mismo país, todos estudiando en esta región. Se encontró que aquellos provenientes de otras regiones presentan menor calidad de vida en SA y PS que los originarios de la Región Metropolitana. Esto nos indica que hay otros factores que influyen en el estado de ánimo de los estudiantes y que son independientes de la latitud.

Una de las limitaciones de este estudio es que el instrumento SPAQ es usado para diagnosticar TAE, el cual puede ser sobreestimado por el cuestionario, por lo tanto, los valores que muestra pueden ser considerados como TAE, cuando en realidad puede ser un síndrome subclínico.

Futuras investigaciones deben considerar otras variables fisiológicas y cognitivas a ser exploradas, como también diferenciales en la percepción de depresión y calidad de vida en distintos períodos estacionales.

\section{Referencias}

1. Ministerio de Salud de Chile. Guía de Práctica Clínica para el tratamiento de la Depresión en Personas Mayores de 15 años. 2013. Disponible en: https://www.minsal. cl/portal/url/item/7222754637c08646e04001011f014e64. pdf.

2. Encuesta Nacional de Salud ENS Chile 2009-2010. Disponible en: https://www.minsal.cl/portal/url/item/ bcb03d7bc28b64dfe040010165012d23.pdf

3. Chen L, Wang L, Qiu XH, Yang XX, Qiao ZX, Yang YJ, et al. Depression among Chinese University Students: 
Depresión en estudiantes de medicina en alta latitud sur - C. Alvarado-Aravena et al

Prevalence and Socio-Demographic Correlates. PLoS One. 2013; 8 (3): 1-6.

4. Bayram N, Bilgel N. The prevalence and socio-demographic correlations of depression, anxiety and stress among a group of university students. Soc Psychiatry Psychiatr Epidemiol. 2008; 43 (8): 667-72.

5. Wong JGWS, Cheung EPT, Chan KKC, Ma KKM, Wa Tang S. Web-Based Survey of Depression, Anxiety and Stress in First-Year Tertiary Education Students in Hong Kong. Aust New Zeal J Psychiatry. 2006; 40 (9): 777-82.

6. Gan GG, Hue YL. Anxiety, depression and quality of life of medical students in Malaysia. Med J Malaysia. 2019; 74 (1): 57-61.

7. Silva V, Costa P, Pereira I, Faria R, Salgueira AP, Costa $\mathrm{MJ}$, et al. Depression in medical students: Insights from a longitudinal study. BMC Med Educ. 2017; 17 (1): 1-9.

8. Rotenstein LS, Ramos MA, Torre M, Bradley Segal J, Peluso MJ, Guille C, et al. Prevalence of depression, depressive symptoms, and suicidal ideation among medical students a systematic review and meta-analysis. JAMA - J Am Med Assoc. 2016; 316 (21): 2214-36.

9. Pinzón-Amado A, Guerrero S, Moreno K, Landínez C, Pinzón J. Ideación suicida en estudiantes de medicina: prevalencia y factores asociados. Rev Colomb Psiquiatr. 2013; 43 (Suppl. 1): 47-55.

10. Irribarra TL, Mery IP, Lira SMJ, Campos DM, González LF, Irarrázaval DS. [Quality of life scores among 411 medical students]. Rev Med Chil. 2018; 146 (11): 1294303.

11. Zhang Y, Qu B, Lun S, Wang D, Guo Y, Liu J. Quality of Life of Medical Students in China: A Study Using the WHOQOL-BREF. PLoS One. 2012; 7 (11).

12. Pagnin D, De Queiroz V. Comparison of quality of life between medical students and young general populations. Educ Heal Chang Learn Pract. 2015; 28 (3): 20912.

13. Rosenthal NE. Seasonal Affective Disorder. Arch Gen Psychiatry. 1984 Jan 1; 41 (1): 72.

14. Jacobsen FM, Wehr TA, Sack DA, James SP, Rosenthal NE. Seasonal affective disorder: A review of the syndrome and its public health implications. Am J Public Health. 1987; 77 (1): 57-60.

15. Wirz-Justice A, Graw P, Kräuchi K, Wacker HR. Seasonality in affective disorders in Switzerland. Acta Psychiatr Scand Suppl. 2003; 108 (418): 92-5.

16. Kasper S, Wehr TA, Bartko JJ, Gaist PA, Rosenthal NE. Epidemiological Findings of Seasonal Changes in Mood and Behavior. Arch Gen Psychiatry. 1989; 46 (9): 823.

17. Goikolea J, Miralles G, Bulbena Cabré A, Vieta E, Bulbe- na A. Adaptación española del Cuestionario de Evaluación de Perfil Estacional (Seasonal Pattern Assessment Questionnaire, SPAQ) en las versiones de adultos e infanto-juvenil. Actas Españolas Psiquiatr. 2003; 31 (4): 192-8.

18. De Rudder B. Grundriss einer Meteorobiologie des Menschen. Springer Berlin Heidelberg 1952.

19. Gatón Moreno MA, González Torres MÁ, Gaviria M. Trastornos afectivos estacionales, "winter blues". Rev Asoc Esp Neuropsiq 2015; 35 (126): 367-80.

20. Magnusson A. Validation of the Seasonal Pattern Assessment Questionnaire (SPAQ). J Affect Disord. 1996; 40 (3): 121-9.

21. Capel Molina JJ, Viedma Muñoz M. Anotaciones geográficas acerca de la insolación en España. Papeles Geogr. 1997; 25: 17-29.

22. Rosen LN, Targum SD, Terman M, Bryant MJ, Hoffman H, Kasper SF, et al. Prevalence of seasonal affective disorder at four latitudes. Psychiatry Res. 1990; 31 (2): 131-44.

23. Ozaki N, Ono Y, Ito A, Rosenthal NE. Prevalence of seasonal difficulties in mood and behavior among Japanese civil servants. Am J Psychiatry. 1995; 152 (8): 1225-7.

24. Sakamoto K, Kamo T, Nakadaira S, Tamura A, Takahashi K. A nationwide survey of seasonal affective disorder at 53 outpatient university clinics in Japan. Acta Psychiatr Scand. 1993; 87 (4): 258-65.

25. J Perdigón AL, Vázquez C. Adaptación española del Inventario para la Depresión de Beck-II ( BDI-II ): 2. Propiedades psicométricas en población general The spanish adaptation of Beck's Depression Inventory-II ( BDI-II ): Clin y Salud 2003; 14 (3): 249-80.

26. Sanz J, Navarro ME. Propiedades psicométricas de una versión española del inventario de ansiedad de beck (BAI) en estudiantes universitarios. [The psychometric properties of a spanish version of the Beck Anxiety Inventory (BAI) in a university students sample]. Ansiedad y Estrés 2003; 9 (1): 59-84.

27. Beck AT, Steer RA. BAI, Beck anxiety inventory: manual. San Antonio (TX, USA.): Psychological Corporation 1993; 23.

28. Puig AA, Natale V, Fabbri M. Propiedades psicométricas de la versión castellana del cuestionario de evaluación de patrón estacional (Seasonal Pattern Assessment Questionnaire, SPAQ). Rev Latinoam Psicol 2006; 38(1): 59-69.

29. Melrose S. Seasonal Affective Disorder: An Overview of Assessment and Treatment Approaches. Depress Res Treat. $2015 ; 2015$.

30. Skevington SM, Lotfy M, O'Connell K A. The World Health Organization's WHOQOL-BREF quality of life 
assessment: Psychometric properties and results of the international field trial A Report from the WHOQOL Group. Qual Life Res. 2004; 13 (2): 299-310.

31. Power M, Harper A, Bullinger M. The World Health Organization WHOQOL-100: Tests of the universality of Quality of Life in 15 different cultural groups worldwide. Health Psychol 199; 18(5): 495-505.

32. Fresno D, Valle J, Pedrejón, Arrauxo A, Benabarre A,
Vieta E. Trastorno afectivo estacional. JANO 2006; 1603: 33.

33. González MM. ¿Cómo la luz "prende" y "apaga" nuestro estado de ánimo. Biblioteca digital. Universidad Católica Argentina. Buenos Aires, 2012.

34. Sam DL. Satisfaction with life among international students: An exploratory study. Social Indicators Research 2001; 53(3): 315-37. 